Research Paper

\title{
The Pathogenesis of Atherosclerosis Based on Human Signaling Networks and Stem Cell Expression Data
}

\author{
Wan Li1 ${ }^{1,}$, Hao Huang1, ${ }^{1,}$, Lei Li ${ }^{2, *}$, Li Wang1 ${ }^{*}$, Yong Li ${ }^{3}$, Yahui Wang ${ }^{1}$, Shanshan Guo ${ }^{1}$, Liansheng Li ${ }^{1}$, \\ Donghua Wang ${ }^{4,}$, Yuehan $\mathrm{He}^{1,}$, , Lina Chen ${ }^{1, \bowtie}$ \\ 1. College of Bioinformatics Science and Technology, Harbin Medical University, Harbin, Heilongjiang, China \\ 2. Department of Neurology, The Second Affiliated Hospital of Harbin Medical University, Harbin, Heilongjiang, China \\ 3. Dean's Office, Harbin Medical University, Harbin, Heilongjiang, China \\ 4. Department of general surgery, General Hospital of Heilongjiang Province Land Reclamation Bureau, 150088, Harbin, China \\ * These authors contributed equally to this work. \\ $\triangle$ Corresponding authors: Lina Chen, E-mail: chenlina@ems.hrbmu.edu.cn; Yuehan He, E-mail: heyuehan56@126.com; Donghua Wang, E-mail: \\ Wangdonghua7885@126.com \\ (C) Ivyspring International Publisher. This is an open access article distributed under the terms of the Creative Commons Attribution (CC BY-NC) license \\ (https://creativecommons.org/licenses/by-nc/4.0/). See http://ivyspring.com/terms for full terms and conditions.
}

Received: 2018.06.15; Accepted: 2018.08.01; Published: 2018.09.07

\begin{abstract}
Atherosclerosis is a common and complex disease, whose morbidity increased significantly. Here, an integrated approach was proposed to elucidate systematically the pathogenesis of atherosclerosis from a systems biology point of view. Two weighted human signaling networks were constructed based on atherosclerosis related gene expression data of stem cells. Then, 37 candidate Atherosclerosis-risk Modules were detected using four kinds of permutation tests. Five Atherosclerosis-risk Modules (three Absent Modules and two Emerging Modules) enriched in functions significantly associated with disease genes were identified and verified to be associated with the maintenance of normal biological process and the pathogenesis and development of atherosclerosis. Especially for Atherosclerosis-risk Emerging Module P96, it could distinguish between normal and disease samples by Supporting Vector Machine with the average expression value of the module as classification feature. These identified modules and their genes may act as potential atherosclerosis biomarkers. Our study would shed light on the signal transduction of atherosclerosis, and provide new insights to its pathogenesis from the perspective of stem cells.
\end{abstract}

Key words: atherosclerosis, stem cell, human signaling network, module, expression data

\section{Introduction}

Atherosclerosis is a chronic inflammatory disease associated with the lipid deposition and plaque fibrosis in the arterial wall, and characterized by marked dysfunction in lipid homeostasis and signaling pathways that control the inflammatory response $[1,2]$.

Stem cells, including endothelial progenitor cells (EPCs), smooth muscle progenitor cells (SMPCs), mesenchymal stem cells (MSCs), different sources of hematopoietic stem cells (HSCs), and adipose-derived stem cells (ADSCs), etc., are cells with self-renewal and differentiation potential. They have been shown to play crucial roles in pathogenesis of atherosclerosis [3]. Undifferentiated HSCs migrate from the bloodstream into diseased tissue and differentiate to macrophages, monocytes, and neutrophils in response to infection and inflammation [4, 5]. Accumulated studies have shown that HSCs are also released into the bloodstream after acute myocardial infarction and augment formation of new monocytes that participate in the progression of atherosclerosis [5]. This indicates that circulating HSCs in the bloodstream represent a pool of undifferentiated stem cells that play a role in the pathogenesis of atherosclerosis. CD34+ stem cells, one kind of HSCs, are localized in atherosclerotic aorta as well as in aortic atherosclerotic regression, and thus could be of great clinical relevance [6]. Medbury et al. examined 
human endarterectomy specimens and found that the fibrous cap of the plaque contained either smooth muscle cells or CD34+ stem cells [7].

The signaling system plays a fundamental part in cells, as it essentially regulates cells and organisms under physiological and pathological states [8]. A malfunction in this system can disrupt the fine-balanced signal transduction significantly, and is believed to be involved in diseases [9]. Inflammatory pathways in stem cells are under stringent control by a variety of transcription factors and coregulatory molecules, such as NF-kB, AP1, the PPAR family, liver $X$ receptor alpha (LXRa/Nr1h3), and their associated coactivators and corepressors [10]. However, the signaling pathways that regulate inflammation and cytokine production in stem cells for atherosclerosis remain incompletely understood. Perturbation of human signaling networks by mutations or abnormal protein expressions underlies the cause of many diseases [11]. It was suggested that diseases could be studied more effectively using systems biology approaches from the perspective of networks [12]. With the increased scope of systems biology, network modules could provide the elucidation of disease mechanisms and provide better strategies for developing multi-marker-module-based risk predictions [13].

Here, we proposed an integrated approach to elucidate systematically the pathogenesis of atherosclerosis by identifying Atherosclerosis-risk Modules based on a human signaling network and disease related gene expression data of stem cells from a systems biology point of view (Figure 1). Our approach would bring a novel understanding of disease signal transduction, and provide valuable insights to the identification of potential biomarkers and the pathogenesis of atherosclerosis.

\section{Materials and methods}

\section{Data source}

A gene expression profile GSE9820 was extracted from the GEO database (http:// www.ncbi.nlm.nih.gov/geo/) [14], containing 23 samples (11 normal samples and 12 atherosclerosis samples) with RNA expressions from CD34+ stem cells. Sixty atherosclerosis genes were extracted from CTD (http://ctdbase.org/) [15], DO (http:// www.disease-ontology.org/) [16] and GWAS (https://www.ebi.ac.uk/gwas/home) [17].

The human signaling network information was derived from Edwin Wang et al. (http:// www.bri.nrc.ca/wang/cancerMap/HumanSignaling Network_v5.txt) [18], which contained 6287 genes and 62239 signaling relations (including activation, inhibition and physical interaction).

\section{Construction of weighted human signaling networks}

Two weighted signaling networks were generated by giving weights to edges of the human signaling network. The weight of the $i$-th edge $E_{i}$ connecting two genes $a$ and $b$ was calculated as Pearson Correlation Coefficient of expression values of $a$ and $b$ in normal and atherosclerosis statuses, respectively.

$$
\operatorname{Weight}\left(E_{i}\right)=\operatorname{Pearson}(a, b)=\frac{\sum\left(a_{j}-\bar{a}\right)\left(b_{j}-\bar{b}\right)}{\left(\sqrt{\sum_{j=1}^{n}\left(a_{j}-\bar{a}\right)^{2}}\right)\left(\sqrt{\sum_{j=1}^{n}\left(b_{j}-\bar{b}\right)^{2}}\right)}
$$

where $a_{j}$ and $b_{j}$ are gene expression values of genes $a$ and $b$ of sample $j, \bar{a}$ and $\bar{b}$ are average expression values for the genes of all samples in each status, and $n$ is the number of samples in corresponding statuses.

Thus, (i) a normal weighted signaling network where correlation coefficients for the normal status were used as edge weights and (ii) an atherosclerosis weighted signaling network where correlation coefficients for the disease status were used as edge weights, were constructed.

\section{Detection of candidate Atherosclerosis-risk Modules}

Candidate Atherosclerosis-risk Modules were detected using two steps.

First, network modules of two weighted signaling networks were mined using the online tool ClusterONE

(http://www.paccanarolab.org.sci-hub.org/clustero ne/), respectively. ClusterONE is a graph-clustering algorithm to identify functional modules in the network. Each module was consisted of a set of genes that were both topologically close and had highly correlated interactions. Modules contained at least four genes were selected.

Next, 4 permutation tests were performed for each network module.

Given a network module $M$ from weighted human signaling networks with $h$ edges $E_{1}, \Lambda, E_{h}$, the expression differential score $V$ was evaluated by differences between Pearson correlation coefficients and those between average expression values, respectively, as follows:

$V(M)=\left\{\begin{array}{c}\sum_{k=1}^{h}\left|S_{k}-S_{k}^{\prime}\right|, \text { Pearson correlation coefficient } \\ \left|S_{u}-S_{u}^{\prime}\right|, \text { Average expression values }\end{array}\right.$

where 


$$
\begin{gathered}
S_{k}=\text { Pearson }(X, Y)=\frac{\sum(X-\bar{X})(Y-\bar{Y})}{\left(\sqrt{\sum_{i=1}^{h}\left(X_{i}-\bar{X}\right)^{2}}\right)\left(\sqrt{\sum_{i=1}^{h}\left(Y_{i}-\bar{Y}\right)^{2}}\right)} \\
S_{k}^{\prime}=\operatorname{Pearson}\left(X^{\prime}, Y^{\prime}\right)=\frac{\sum\left(X^{\prime}-\bar{X}^{\prime}\right)\left(Y^{\prime}-\bar{Y}^{\prime}\right)}{\left(\sqrt{\sum_{i=1}^{h}\left(X_{i}^{\prime}-\bar{X}^{\prime}\right)^{2}}\right)\left(\sqrt{\sum_{i=1}^{h}\left(Y_{i}^{\prime}-\bar{Y}^{\prime}\right)^{2}}\right)} \\
S_{u}=\text { Expression }(X)=\frac{1}{g} \sum_{i=1}^{g} X_{i} \\
S_{u}^{\prime}=\text { Expression }\left(X^{\prime}\right)=\frac{1}{g} \sum_{i=1}^{g} X_{i}^{\prime}
\end{gathered}
$$

For normal and atherosclerosis samples, $X, Y$ and $X^{\prime}, Y^{\prime}$ are gene expression values, $S_{k}$ and $S^{\prime}{ }_{k}$ are Pearson correlation coefficients of the k-th edge , $S_{u}$ and $S^{\prime}{ }_{u}$ are the average expression value of genes in $M$, respectively. $g$ is the number of genes in the module. For each network module, the differential score $V$ was calculated.

To obtain the significance of each module, four permutation tests were performed. From weighted human signaling networks, 1000 degree-conserved random modules and 1000 size-conserved random modules were constructed for each module. Random differential scores $V_{1}, \Lambda, V_{1000}$ for Pearson correlation coefficients and average expression values of random modules were calculated, respectively. Modules with expression differential scores significantly greater than the random ones (permutation test, FDR $p<$ 0.05 ) were considered significant. A module significant in at least 3 of 4 permutation tests was a candidate Atherosclerosis-risk module.

\section{Identification of Atherosclerosis-risk Modules}

Candidate Atherosclerosis-risk Modules that were enriched in functions significantly associated with atherosclerosis genes were defined as Atherosclerosis-risk Modules. The functional annotation analysis was performed with atherosclerosis genes or genes in each candidate Atherosclerosis-risk Modules using the hyper-geometric test:

$$
P(X=1)=\frac{C_{m}^{l} C_{q-m}^{g-l}}{C_{q}^{g}}
$$

where $q$ denotes the number of all human genes, $g$ denotes the number of atherosclerosis genes or genes in candidate Atherosclerosis-risk Modules, $m$ denotes the number of genes in function $j, l$ denotes the number of genes of Atherosclerosis-risk Modules in function $j$. The Bonferroni-corrected p-value $<0.05$ was set as the criterion for screening Atherosclerosis-risk Modules.

\section{Results}

\section{Atherosclerosis-risk Modules}

Using four kinds of permutation tests, 37 candidate Atherosclerosis-risk Modules significantly differential between normal and atherosclerosis statuses were detected from two weighted human signaling networks. After functional enrichment analysis, 5 Atherosclerosis-risk Modules enriched in functions significantly associated with atherosclerosis genes were identified (Table 1). Among them, 3 Atherosclerosis-risk Modules (C83, C368, C377) which were identified from the normal weighted signaling network were defined as Atherosclerosis-risk Absent Modules, and the other 2 modules (P96, P20) which were identified from the atherosclerosis weighted signaling network were defined as Atherosclerosisrisk Emerging Modules.

Table 1. Atherosclerosis-risk Modules.

\begin{tabular}{lll}
\hline $\begin{array}{l}\text { Atherosclerosis-risk } \\
\text { Module }\end{array}$ & $\begin{array}{l}\text { Number of } \\
\text { genes }\end{array}$ & Genes of Atherosclerosis-risk Modules \\
\hline C83 & 8 & $\begin{array}{l}\text { DDB2, ERCC5, TAF1, ERCC3, SMARCC2, } \\
\text { SMARCD1, CIITA, SMARCA4 } \\
\text { CX3CR1, ARRB2, CCR1, CCL7, CCR2, } \\
\text { C368 }\end{array}$ \\
CXCL16, CXCL3, CCL8, CXCL1, ADRBK2 \\
C377 & 10 & $\begin{array}{l}\text { LYN, RGS16, INPP5D, SYK, CD79B, CD79A, } \\
\text { BLK, PLA2G4A, CD22, LAT2, FCGR2B, }\end{array}$ \\
P20 & 16 & $\begin{array}{l}\text { PPP1R8, PTPN6, LIMS1, NCK2, PDGFB } \\
\text { BUB1B, CDC23, PSMD1, CDC20, CCNB1, } \\
\text { BUB1, CCNB2, ANAPC7, MAD2L1, UBE2E1, } \\
\text { PSMD14, PSMA5 }\end{array}$ \\
P96 & 12 & $\begin{array}{l}\text { IL1A, IL6, FAS, CXCL1, IL8, IL1B, ICAM1, } \\
\text { ADIPOQ }\end{array}$ \\
\hline
\end{tabular}

\section{Atherosclerosis related function analysis of Atherosclerosis-risk Modules}

Atherosclerosis-risk Modules were significantly enriched in functional categories and pathways related to atherosclerosis (Table S1). Atherosclerosis-risk Module C368 was significantly enriched in functions including "GO:0005125 cytokine activity", "GO:0006955 immune response", "GO:0006954 inflammatory response" and so on. Atherosclerosis-risk Module C377 was significantly enriched in "GO:0002684 positive regulation of immune system process" and other functions. Atherosclerosis-risk Module P96 was significantly enriched in a number of functions, such as "58.(CD40L)_immnosurveillance".

Functions "GO:0006954 inflammatory response", "GO:0006955 immune response", "GO:00026 $84 \sim$ positive regulation of immune system process" and "58.(CD40L)_immnosurveillance" were associated with the immune system and inflammatory. Atherosclerosis is an inflammatory disease with lesions filling with immune cells that can orchestrate and affect inflammatory responses. 
Unstable plaques were particularly rich in activated immune cells, suggesting that they might initiate plaque activation [19]. "GO:0005125 cytokine activity" is related to the cytokines. Many cytokines are expressed in atherosclerotic plaques and all cells involved in the disease are capable of producing cytokines and responding to them. Cytokines could modulate endothelial cells (EC) permeability.
Activated ECs release a range of chemokines and other cytokines that then cause the recruitment of circulating immune cells, particularly monocytes and T lymphocytes [20, 21]. In addition, the ECs express adhesion proteins, such as intercellular adhesion molecule-1 (ICAM1) and vascular cell adhesion molecule-1 (VCAM1), which participate in the recruitment of immune cells [22, 23].
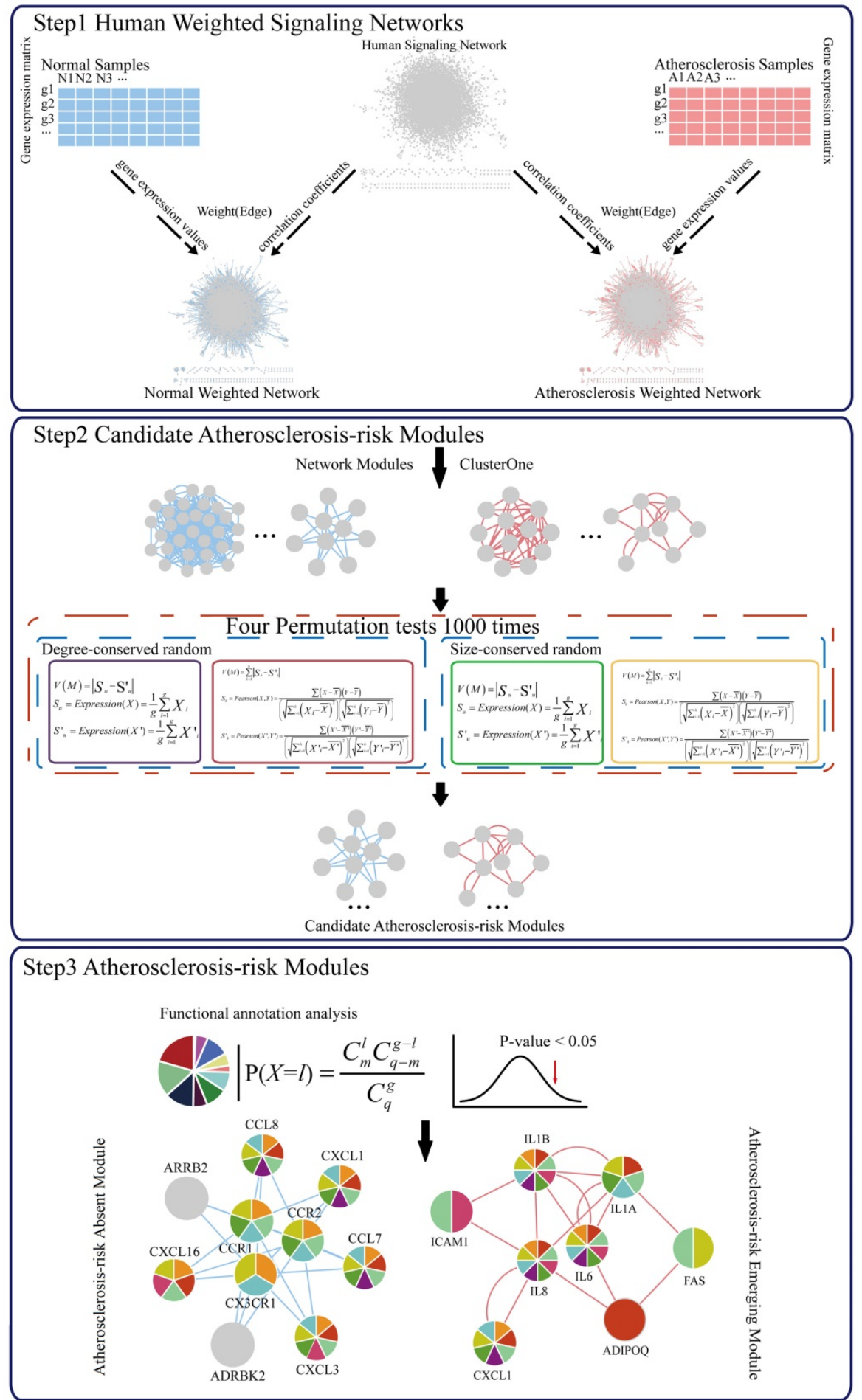

Figure 1: A schematic diagram of Atherosclerosis-risk Modules identification. Atherosclerosis-risk Modules were identified from the systems level based on weighted human signaling networks and atherosclerosis related stem cell expression profiles. A) Construction of weighted human signaling networks. B) Detection of candidate Atherosclerosis-risk Modules. C) Identification of Atherosclerosis-risk Modules. 
Atherosclerosis-risk Modules, especially Atherosclerosis-risk Absent Modules, were significantly enriched in the functional categories related to the basic functions of body, such as "GO:0031328 positive regulation of cellular biosynthetic process", "GO:0051173 positive regulation of nitrogen compound metabolic process", "GO:0010604 positive regulation of macromolecule metabolic process" and "GO:0031399 regulation of protein modification process". These functions are crucial to maintain the normal biological processes, and disturbance and changes of them were associated with atherosclerosis closely [24-27].

Atherosclerosis-risk Absent Modules and Atherosclerosis-risk Emerging Modules were significantly enriched in functional categories significantly related to the disease, such as inflammatory response and cytokine activity, which suggested that these modules play important roles in the pathogenesis and development of atherosclerosis.

\section{Analysis of genes in Atherosclerosis-risk Modules}

The genes in Atherosclerosis-risk Modules were linked closely with the pathogenesis of atherosclerosis as they possessed disease associated functions. In three Atherosclerosis-risk Absent Modules, C368, C 377 and C83, $80 \%, 68.8 \%$ and $50 \%$ of genes were verified by literature to be associated with the pathogenesis of atherosclerosis, respectively. In two Atherosclerosis-risk Emerging Modules, P96 and P20, $100 \%$ and $50 \%$ of genes were verified to be associated with the pathogenesis of atherosclerosis, respectively. It was worth noting that genes in Atherosclerosis-risk Absent Module C368 and Atherosclerosis-risk Emerging Module P96 with high verification percent were both enriched in functional categories associated with atherosclerosis (Figure 2), such as
"GO:0009611 response to wounding", "GO:0006954 inflammatory response", "GO:0006955 immune response" and "GO:0005125 cytokine activity". In addition, some categories, such as "GO:0042330 taxis", "GO:0006935 chemotaxis" and "hsa04060: Cytokine-cytokine receptor interaction", in which known atherosclerosis genes could not be significantly enriched, were significantly enriched for Atherosclerosis-risk Absent Module C368 and Atherosclerosis-risk Emerging Module P96. These categories have also been proven to be associated with atherosclerosis closely. "GO:0042330 taxis" and "GO:0006935 chemotaxis" were associated with chemotaxis, which recruited monocyte and macrophages into the subendothelial space where they phagocytized oxidized lipids, formed foam cells and initiated processes leading to advanced lesions $[28,29]$.

Atherosclerosis-risk Absent Module C368 was identified only from the normal weighted signaling network rather than the atherosclerosis weighted signaling network. Most genes in this module were members of chemokines. These chemokines were considered to be pro-inflammatory and could be induced during an immune response to recruit cells of the immune system to a site of infection [30]. Meanwhile, chemokines were also considered to be homeostatic and involved in controlling the migration of cells during normal processes of tissue maintenance or development and could promote wound healing [31, 32]. For instance, CCR1 was confirmed to play a protective role on atherosclerosis [29]. CX3CR1 is the only member of CX3C chemokines Fractalkine (FKN) receptor [33]. Resent research has shown that FKN / CX3CR1 was involved in the inflammatory process and played a pro-inflammatory effect of atherosclerosis [2, 33]. Hence, absence of Atherosclerosis-risk Module C368
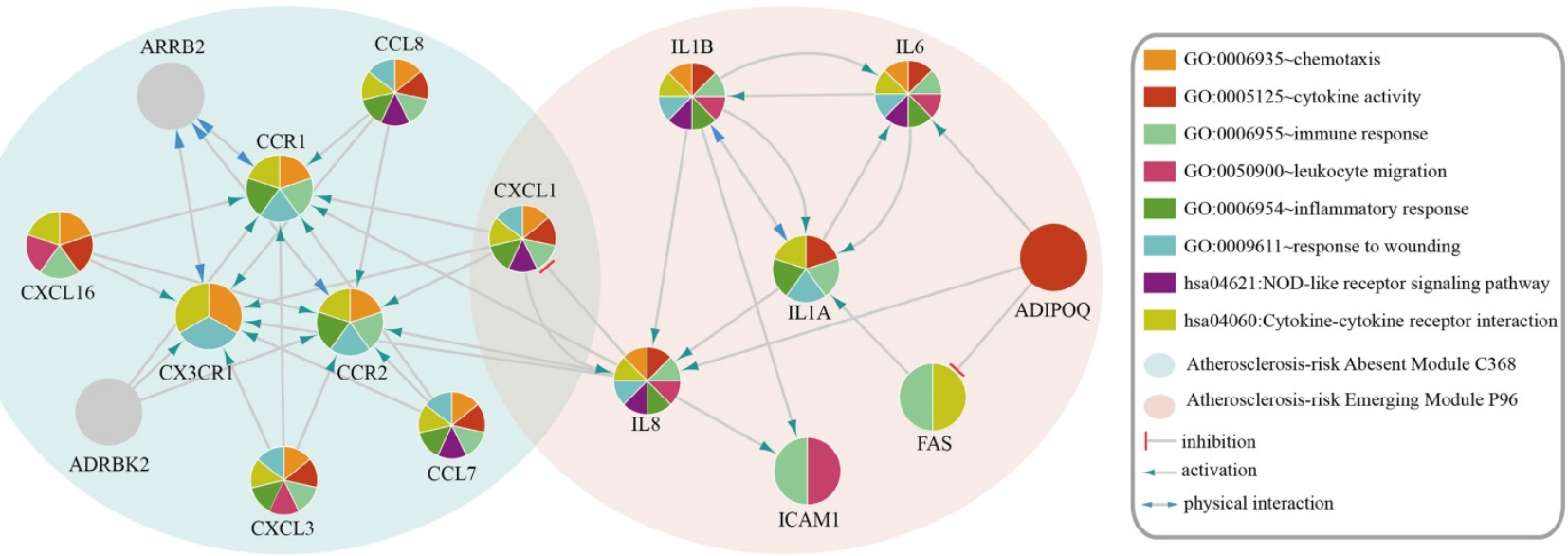

Figure 2: Functional categories of Atherosclerosis-risk Absent Module C368 and Atherosclerosis-risk Emerging Module P96. Different patterns of each circle represent different functions these modules enriched in. 
in disease status suggested that the protective effect of Module C368 were suppressed, which promoted the occurrence of atherosclerosis.

Interestingly, CXCL1 was in both Atherosclerosis-risk Absent Module C368 and Emerging Module P96. CXCL1 is an antimicrobial gene encodes a member of the CXC subfamily of chemokines. This protein played a role in inflammation as a chemoattractant for neutrophils and could increase chemotactic activity [34]. The appropriate expression of CXCL1 would inhibit the formation of plaques [34, 35]. Expression difference of CXCL1 in two modules demonstrated the function changes in the process of atherosclerosis.

Moreover, Atherosclerosis-risk Emerging Module P96 could be identified only in atherosclerosis weighted signaling network, and genes in this module were mainly related to inflammatory response and chemotaxis. For instance, IL1A and IL1B belonged to interleukin family, which attracted neutrophils, and caused the release of inflammatory mediators. IL1B was involved in the pathogenesis of atherosclerosis for endothelial cells. Secreted IL1A, IL1B amplified and differentiated progenitor cells (CD34 (+)) cells via c-MSCs signal [22, 36-38]. IL6 could lead to the accumulation of inflammatory cells in atherosclerotic plaques, affect the stability of plaque and promote thrombosis and necrosis [39, 40]. IL8 is the main attractor and activator of neutrophils. IL8 also had a role in promoting artery atherosclerotic plaque pathological angiogenesis, which was one of the main factors that lead to plaque instability $[22,41]$. Hence, the emergence of Atherosclerosis-risk Module P96 in disease status may facilitate the development of atherosclerosis.

\section{Discussion}

Atherosclerosis is a disease of the arteries characterized by the deposition of plaques of fatty material on their inner walls. Compelling evidence has demonstrated how risk factors, such as hypercholesterolemia, provoked inflammation and reinforced the initiation and progression of atherosclerosis [42]. Interestingly, recent studies have reported that hypercholesterolemia had an impact on stem cells to strengthen inflammation $[42,43]$. Studies have shown that atherosclerotic plaques were closely associated with ectopic calcification of vascular differentiation of stem cells [44]. Moreover, perturbation of the signals stem cells secreted by mutations or abnormal protein expression underlies a cause of atherosclerosis $[45,46]$. Here, we put forward an integrated approach to identify Atherosclerosis-risk Modules using atherosclerosis related stem cell expression profiles based on a human signaling network. Five Atherosclerosis-risk Modules (three Absent Modules and two Emerging Modules) were identified from two weighted human signaling networks, which were both significantly differential between normal and disease statuses and enriched in functions significantly associated with atherosclerosis genes.

Atherosclerosis-risk Absent Modules were only identified from the normal weighted signaling network, which were crucial to maintain the normal cell regulation and transport function. Absence of these modules in disease status would lead to the disorder of the signal of stem cells and the change of the internal environment so as to participate in the pathogenesis of atherosclerosis [24-27, 47, 48]. The 3 Atherosclerosis-risk Absent Modules enriched significantly in the functional categories related to the maintenance of normal biological process, including "GO:0031328 positive regulation of cellular biosynthetic process", "GO:0051173 positive regulation of nitrogen compound metabolic process" and "GO:0006935 chemotaxis". Cellular biosynthetic process is involved in many biological processes. DeBerardinis et al. have demonstrated that the disorder of cellular biosynthetic process could lead to related signal disorders and the development of diseases [49]. Qiao et al. have proved that metabolic disorders amplified macrophage chemotactic responses and accelerated the atherogenesis process [50]. The production of reactive nitrogen species was implicated in atherosclerosis principally as means of damaging low-density lipoprotein that in turn initiated the accumulation of cholesterol [51]. Meanwhile, the average expression value of Atherosclerosis-risk Absent Modules in disease status was lower than that in normal status significantly, which suggested that the absence of Atherosclerosis-risk Absent Modules might involve in the pathogenesis of atherosclerosis.

Atherosclerosis-risk Emerging Modules were only identified in atherosclerosis weighted signaling network, which were associated with its pathogenesis, especially for Atherosclerosis-risk Module P96. It was enriched significantly in functional categories related to the pathogenesis of atherosclerosis. Meanwhile, the average expression value of Atherosclerosis-risk Emerging Module P96 in atherosclerosis status was higher than that in normal status significantly. Moreover, the ability to classify samples of normal and disease statuses could reveal the association with diseases using Supporting Vector Machine (SVM) or random forest [52]. Here, SVM was applied to classify samples of different statuses (normal/atherosclerosis) of the stem cell expression profile GSE9820 with the average expression value of Atherosclerosis-risk 
Emerging Module P96 as classification feature, and Leave-one-out cross-validation was performed [53]. A receiver operating characteristic (ROC) curve was plotted and the area under the curve (AUC) was 0.841. It demonstrated that Atherosclerosis-risk Emerging Module P96 had a good classification performance in distinguishing between normal and disease samples. Meanwhile, all genes of Atherosclerosis-risk Emerging Module P96 were proven to be associated with the pathogenesis of atherosclerosis proven by literature. Three genes, IL6, ICAM1 and ADIPOQ, were confirmed to be atherosclerosis genes by databases CTD, DO and GWAS. ADIPOQ, as the start point of signal transduction in Module P96, regulated many processes and affected other genes in the module. ICAM1 might influence genes outside the module since it was the end of signals. IL6 and other interleukins in this module all regulated other genes or were regulated by other genes in several ways. Hence, the emergence and activation of Atherosclerosis-risk Emerging Module or dysfunction of its genes could contribute to the development of atherosclerosis, and the genes of Atherosclerosis-risk Emerging Module P96 might be potential disease genes.

Though sample size (11 normal and 12 atherosclerosis samples) of the expression profile we used could be a limitation of our study, it was still enough to construct weighted human signaling networks since they were significantly consistent in expression $(p<0.05)$. From these networks, candidate Atherosclerosis-risk Modules were detected and Atherosclerosis-risk Modules were further identified. Larger sample size might help to improve the results and illustrate the pathogenesis of atherosclerosis better in the future.

In summary, a novel integrated approach was proposed to identify Atherosclerosis-risk Modules from the systems level based on weighted human signaling networks and atherosclerosis related stem cell expression profiles. Five Atherosclerosis-risk Modules (three Absent Modules and two Emerging Modules) were identified and verified to be associated with the pathogenesis of atherosclerosis. Atherosclerosis-risk Emerging Module P96 could distinguish between normal and disease samples. These modules and their genes may act as potential atherosclerosis biomarkers. Our study would shed light on the signal transduction of atherosclerosis, and provide new insights to its pathogenesis from the perspective of stem cells.

\section{Supplementary Material}

Supplementary table.

http://www.ijbs.com/v14p1678s1.pdf

\section{Acknowledgements}

This work was supported in part by the National Natural Science Foundation of China [grant numbers 61702141 and 61272388]; the Health and Family Planning Commission Scientific Research Subject of Heilongjiang Province [grant number 2016-203]; the Fundamental Research Funds for the Provincial Universities in Heilongjiang Province [grant number 2017-KYYWF-0303] (the Innovative Scientific Research Funding Project of Harbin Medical University [grant number 2017JCZX46]); the Heilongjiang Postdoctoral Funds for Scientific Research Initiation [grant number LBH-Q17132]; the University Student Innovation and Entrepreneurship Training Program in Heilongiiang Province [grant numbers 201710226011 and 201710226025]; and the

Harbin Applied Technology Research and Development Project [grant number 2016RQQXJ105].

\section{Competing Interests}

The authors have declared that no competing interest exists.

\section{References}

1. Buckley ML, Ramji DP. The influence of dysfunctional signaling and lipid homeostasis in mediating the inflammatory responses during atherosclerosis. Biochimica et biophysica acta. 2015; 1852: 1498-510.

2. Galkina E, Ley K. Immune and inflammatory mechanisms of atherosclerosis $\left({ }^{*}\right)$. Annu Rev Immunol. 2009; 27: 165-97.

3. Orlandi A. The contribution of resident vascular stem cells to arterial pathology. Int J Stem Cells. 2015; 8: 9-17.

4. Shi C, Pamer EG. Monocyte recruitment during infection and inflammation. Nat Rev Immunol. 2011; 11: 762-74.

5. Cimato TR, Palka BA, Lang JK, et al. LDL cholesterol modulates human CD34+ HSPCs through effects on proliferation and the IL-17 G-CSF axis. PLoS One. 2013; 8: e73861.

6. Zenovich AG, Taylor DA. Atherosclerosis as a disease of failed endogenous repair. Front Biosci. 2008; 13: 3621-36.

7. Medbury HJ, Tarran SL, Guiffre AK, et al. Monocytes contribute to the atherosclerotic cap by transformation into fibrocytes. Int Angiol. 2008; 27: 114-23.

8. Xie $\mathrm{R}$, Huang $\mathrm{H}, \mathrm{Li} \mathrm{W}$, et al. Identifying progression related disease risk modules based on the human subcellular signaling networks. Mol Biosyst. 2014; 10: 3298-309.

9. Mencarelli C, Martinez-Martinez P. Ceramide function in the brain: when a slight tilt is enough. Cell Mol Life Sci. 2013; 70: 181-203.

10. Rahman SM, Baquero KC, Choudhury M, et al. C/EBPbeta in bone marrow is essential for diet induced inflammation, cholesterol balance, and atherosclerosis. Atherosclerosis. 2016; 250: 172-9.

11. Brognard J, Hunter T. Protein kinase signaling networks in cancer. Curr Opin Genet Dev. 2011; 21: 4-11.

12. Zhu L, Su F, Xu Y, et al. Network-based method for mining novel HPV infection related genes using random walk with restart algorithm. Biochimica et biophysica acta. 2018; 1864: 2376-83.

13. Gustafsson $\mathrm{M}$, Nestor $\mathrm{CE}$, Zhang $\mathrm{H}$, et al. Modules, networks and systems medicine for understanding disease and aiding diagnosis. Genome Med. 2014; 6: 82 .

14. Barrett $\mathrm{T}$, Wilhite SE, Ledoux $\mathrm{P}$, et al. NCBI GEO: archive for functional genomics data sets--update. Nucleic acids research. 2013; 41: D991-5.

15. Davis AP, Grondin CJ, Lennon-Hopkins K, et al. The Comparative Toxicogenomics Database's 10th year anniversary: update 2015. Nucleic Acids Res. 2015; 43: D914-20.

16. Schriml LM, Arze C, Nadendla S, et al. Disease Ontology: a backbone for disease semantic integration. Nucleic acids research. 2012; 40: D940-6.

17. Welter D, MacArthur J, Morales J, et al. The NHGRI GWAS Catalog, a curated resource of SNP-trait associations. Nucleic Acids Res. 2014; 42: D1001-6.

18. Cui Q, Ma Y, Jaramillo M, et al. A map of human cancer signaling. Mol Syst Biol. 2007; 3: 152.

19. Hansson GK. Regulation of immune mechanisms in atherosclerosis. Ann N Y Acad Sci. 2001; 947: 157-65; discussion 65-6. 
20. Ramji DP, Davies TS. Cytokines in atherosclerosis: Key players in all stages of disease and promising therapeutic targets. Cytokine Growth Factor Rev. 2015; 26: 673-85.

21. Bhargava $\mathrm{P}$, Lee $\mathrm{CH}$. Role and function of macrophages in the metabolic syndrome. Biochem J. 2012; 442: 253-62.

22. Sprague AH, Khalil RA. Inflammatory cytokines in vascular dysfunction and vascular disease. Biochem Pharmacol. 2009; 78: 539-52.

23. Pober JS. Endothelial activation: intracellular signaling pathways. Arthritis Res. 2002; 4 Suppl 3: S109-16.

24. Shibata N, Glass CK. Regulation of macrophage function in inflammation and atherosclerosis. J Lipid Res. 2009; 50 Suppl: S277-81.

25. Schlebusch M, Forchhammer K. Requirement of the nitrogen starvation-induced protein $\mathrm{Sll} 0783$ for polyhydroxybutyrate accumulation in Synechocystis sp. strain PCC 6803. Appl Environ Microbiol. 2010; 76: 6101-7.

26. Baenke F, Dubuis S, Brault C, et al. Functional screening identifies MCT4 as a key regulator of breast cancer cell metabolism and survival. J Pathol. 2015; 237: 152-65.

27. Migliaccio N, Martucci NM, Ruggiero I, et al. Ser/Thr kinases and polyamines in the regulation of non-canonical functions of elongation factor 1A. Amino Acids. 2016

28. Singh RB, Mengi $\mathrm{SA}, \mathrm{Xu} \mathrm{YJ}$, et al. Pathogenesis of atherosclerosis: A multifactorial process. Exp Clin Cardiol. 2002; 7: 40-53.

29. Braunersreuther V, Zernecke A, Arnaud C, et al. Ccr5 but not Ccr1 deficiency reduces development of diet-induced atherosclerosis in mice. Arterioscler Thromb Vasc Biol. 2007; 27: 373-9.

30. Cekici A, Kantarci A, Hasturk H, et al. Inflammatory and immune pathways in the pathogenesis of periodontal disease. Periodontol 2000. 2014; 64: 57-80.

31. Martins-Green M, Petreaca M, Wang L. Chemokines and Their Receptors Are Key Players in the Orchestra That Regulates Wound Healing. Adv Wound Care (New Rochelle). 2013; 2: 327-47.

32. Loffek S, Schilling O, Franzke CW. Series "matrix metalloproteinases in lung health and disease": Biological role of matrix metalloproteinases: a critical balance. Eur Respir J. 2011; 38: 191-208.

33. Stolla M, Pelisek J, von Bruhl ML, et al. Fractalkine is expressed in early and advanced atherosclerotic lesions and supports monocyte recruitment via CX3CR1. PLoS One. 2012; 7: e43572.

34. Turner MD, Nedjai B, Hurst T, et al. Cytokines and chemokines: At the crossroads of cell signalling and inflammatory disease. Biochimica et biophysica acta. 2014; 1843: 2563-82.

35. Hol J, Otterdal K, Breland UM, et al. Statins affect the presentation of endothelial chemokines by targeting to multivesicular bodies. PLoS One. 2012; 7: e40673.

36. Imai $\mathrm{Y}$, Dobrian AD, Weaver JR, et al. Interaction between cytokines and inflammatory cells in islet dysfunction, insulin resistance and vascular disease. Diabetes Obes Metab. 2013; 15 Suppl 3: 117-29.

37. Bartosh TJ, Ylostalo JH, Bazhanov N, et al. Dynamic compaction of human mesenchymal stem/precursor cells into spheres self-activates caspase-dependent IL1 signaling to enhance secretion of modulators of inflammation and immunity (PGE2, TSG6, and STC1). Stem Cells. 2013; 31: 2443-56.

38. Wakita D, Kurashima Y, Crother TR, et al. Role of Interleukin-1 Signaling in a Mouse Model of Kawasaki Disease-Associated Abdominal Aortic Aneurysm. Arterioscler Thromb Vasc Biol. 2016; 36: 886-97.

39. Wang SS, Hu SW, Zhang QH, et al. Mesenchymal Stem Cells Stabilize Atherosclerotic Vulnerable Plaque by Anti-Inflammatory Properties. PLoS One. 2015; 10: e0136026.

40. Kleemann R, Zadelaar S, Kooistra T. Cytokines and atherosclerosis: a comprehensive review of studies in mice. Cardiovasc Res. 2008; 79:360-76.

41. Papadopoulou C, Corrigall V, Taylor PR, et al. The role of the chemokines MCP-1, GRO-alpha, IL-8 and their receptors in the adhesion of monocytic cells to human atherosclerotic plaques. Cytokine. 2008; 43: 181-6.

42. Ma X, Feng Y. Hypercholesterolemia Tunes Hematopoietic Stem/Progenitor Cells for Inflammation and Atherosclerosis. Int J Mol Sci. 2016; 17.

43. Tie G, Messina KE, Yan J, et al. Hypercholesterolemia induces oxidant stress that accelerates the ageing of hematopoietic stem cells. J Am Heart Assoc. 2014; 3: e000241.

44. Leszczynska A, O'Doherty A, Farrell E, et al. Differentiation of Vascular Stem Cells Contributes to Ectopic Calcification of Atherosclerotic Plaque. Stem Cells. 2016; 34: 913-23.

45. Johnstone SE, Baylin SB. Stress and the epigenetic landscape: a link to the pathobiology of human diseases? Nat Rev Genet. 2010; 11: 806-12.

46. Andres V, Gonzalez JM. Role of A-type lamins in signaling, transcription, and chromatin organization. J Cell Biol. 2009; 187: 945-57.

47. Nagy EE, Varga-Fekete T, Puskas A, et al. High circulating osteoprotegerin levels are associated with non-zero blood groups. BMC Cardiovasc Disord. 2016; 16: 106.

48. Christ A, Bekkering S, Latz E, et al. Long-term activation of the innate immune system in atherosclerosis. Semin Immunol. 2016; 28: 384-93.

49. Wellen KE, Thompson CB. Cellular metabolic stress: considering how cells respond to nutrient excess. Mol Cell. 2010; 40: 323-32.

50. Qiao $M$, Zhao $Q$, Lee $C F$, et al. Thiol oxidative stress induced by metabolic disorders amplifies macrophage chemotactic responses and accelerates atherogenesis and kidney injury in LDL receptor-deficient mice. Arterioscler Thromb Vasc Biol. 2009; 29: 1779-86.
51. Patel RP, Moellering D, Murphy-Ullrich J, et al. Cell signaling by reactive nitrogen and oxygen species in atherosclerosis. Free Radic Biol Med. 2000; 28: 1780-94.

52. Tang W, Wan S, Yang Z, et al. Tumor origin detection with tissue-specific miRNA and DNA methylation markers. Bioinformatics. 2018; 34: 398-406.

53. Witten IH, Frank E. Data Mining: Practical machine learning tools and techniques. 2005 